\title{
Cutting-Edge Robotic Intravenous Pole: Preliminary Design and Survey in Academic Medical Center in Lebanon
}

\author{
Abbas Sayed-Kassem ${ }^{1}$, Ali Ghandour ${ }^{1}$, Lara Hamawy ${ }^{1}$ and Amira J. Zaylaa ${ }^{1,2 *}$ \\ ${ }^{1}$ Department of Biomedical Engineering, School of Engineering, Lebanese International University, Lebanon \\ ${ }^{2}$ Neuroscience Research Center, Faculty of Medical Sciences, Lebanese University, Lebanon
}

\begin{abstract}
Intravenous poles are biomedical healthcare supportive tools that aide in holding and delivering medications to patients through intravenous injections. Intravenous infusion relies on the weight of the Intravenous tubing fluid. Continuous follow-up of the intravenous bag is required to replace the fluid when emptied. Also, mobilization of the intravenous pole is required from patients and nurses. This leads to discomfort and inconvenience for both patients and hospital staff. Despite the existing intravenous poles have solved their difficulty, however, they were either not feasible, or couldn't hold a lot of weight in the bag. Moreover, they lack any alarm system which indicates an empty bag. To improve current intravenous poles, we aim to develop a cutting-edge robotic intravenous pole mounted with an alarm system. The robotic intravenous pole comprises motors to hold large weights, sensors and wireless joystick technology. Experimental results dedicated to the design showed that the novel designed pole mobilization is improved by the use of stepper motors, omni wheels, micro-controller, and a joystick. Also, the alarm system has added alert to patients and nurses when the intravenous bags were emptied. Moreover, the robotic intravenous pole moved in all directions and rotated, with a press of a button. This design surpasses the movements and weight tolerance provided by alternative designs, and it is mechanically and electrically safe on the patients. Survey results highlighted the acceptance and motivation of having and using and Robotic IV pole as compared to alternatives.
\end{abstract}

Keywords: Intravenous poles; Novel robotic intravenous poles; Alarm system; Novel technology; Healthcare application; Survey

\section{Introduction}

Intravenous (IV) poles are medical supportive tools widely utilized in healthcare practices. IV poles carry bags and deliver the necessary medications to patients. IV medication are introduced to the patient through an IV injection [1,2]. All IV pole designs are apparently similar with hook at the top and wheels at the base. These designs differ by the accessories attached to them $[3,4]$. Despite the prominence use of IV poles, they were subject to a slight improvement over the past decades [1-6].

Research studies and practices reported that one of the basic shortcomings of IV poles available in the market is their difficulty to mobilize freely. When patients want to move beyond a confined area, they need to drag their own IV pole or get the assist from the nurse. Accordingly, the nurse will consume time accompanying the patient and dragging the IV stand. The former and latter steps are inconvenient for both the patient and the nurse [7-9].

To overcome the IV poles which arise in daily medical practices of elderly patients, Wiederhold et al. proposed several general robotic solutions for patient's in their every-day's activities inside and outside hospitals [10]. This thing aided in augmented patient's acceptance of an assistant robotic system was enhanced [10].

Moreover, with the advancement of the healthcare sector, Qureshi et al. introduced robots in the day-to-day operations. Also, the healthcare sector is encouraging the human resources to adopt robotic systems, train them and get their feedback [8]. Later, Binger et al. have proposed an autonomous IV stand, however, the system was not mounted with an alarm system for detecting the IV bag medication level and the design couldn't handle common medical equipment such as: oxygen tanks, infusion pumps, etc [9].

With the progress of robot-based healthcare services, there is no optimal IV pole design that fully takes into consideration the comfort of both patients and nurses. Patients, in particularly, those at young and elderly ages, or those who undergo chemotherapy find difficulty in dragging current IV poles in the market [3,4,11]. Furthermore, most of the current IV stands does not contain an intrinsic alarm which alert both the patient and nurse when the whole fluid in the bag is emptied [4]. In this province, questions arise: can a new design overcome the IV stands shortcomings? Can an ovel biomedical instrument be, mounted by an alarm system, with stand a large weight and launched remotely by a press of a button? To match the patients', need and to improve current IV pole designs, we carry out this project. Our objective is to design a new Robotic IV pole and validate its performance. We aim to launch the new design through a press of a button, and benefit from its unique mobility, weight, and alarm system. We propose to equip the Robotic IV pole with an intrinsic/embedded alarm system. Moreover, since there are several types of IV stands that are currently available; we aim to compare it to alternative IV stands in market. Then ovel robotic IV pole has to comprise a transmitted side, and a receiving side in order to provide a remote communication and comfort not only to patients but also to nurses. The safety is considered and the price is reasonable relative to its quality. The remainder of this paper is organized as follows. In section 2, we provide the existing IV pole designs. In sections 3, we introduce a detailed description of our novel robotic IV pole from the design to the implementation reaching to the

*Corresponding author: Amira J. Zaylaa, Department of Biomedical Engineering School of Engineering, Neuroscience Research Center, Faculty of Medical Sciences, Lebanese International University, Lebanon, Lebanese University, Lebanon, Tel: 00913940869; E-mail: amira.zaylaa2@ul.edu.lb

Received May 28, 2017; Accepted June 13, 2017; Published June 19, 2017

Citation: Sayed-Kassem A, Ghandour A, Hamawy L, Zaylaa AJ (2017) CuttingEdge Robotic Intravenous Pole: Preliminary Design and Survey in Academic Medical Center in Lebanon. J Biomed Eng Med Devic 2: 124. doi: 10.4172/24757586.1000124

Copyright: ㄷ 2017 Sayed-Kassem A, et al. This is an open-access article distributed under the terms of the Creative Commons Attribution License, which permits unrestricted use, distribution, and reproduction in any medium, provided the original author and source are credited. 
safety measures. In section 4 , we set out the experimental results. In section 5 we showcase the survey requirements and results. In section 6 we discuss the results, provide a general conclusion and finally in section 7 we give our recommendations to improve the novel robot.

\section{Intravenous Poles Existing}

Herein we provide an overview of IV poles that exit in developed and non-developed countries [4]. Figure 1 showcases different IV poles existing in the market and under research and development. The existing IV poles are, but not limited to, Figure 1 (a) and Figure 1 (b) the simple free standing IV pole, Figure 1(c) Dyuan IV pole, Figure 1 (d) Hanging IV pole, Figure 1 (e) Lakeside chrome infusion pump pole and Figure 1 (f) other features of the infusion pump IV pole [4].

\section{Simple free standing intravenous poles}

The most common type of IV stand is the free-standing mobile pole as shown in Figure 1 (a) and Figure 1 (b). This design has been modified to facilitate intravenous feeding during transportation of patients [7]. All designs of this type are similar however they include improvements in hangers and accessories [12-19]. The primary difference is in the design is the base, some enhanced forms include support for the legs of the stand [20]. Their height is adjustable by loosening the adjustment screw and sliding the upper tube up or down. Stands of this design are usually on casters which make them mobilizes feasibly. This gives the patient the opportunity to get out of bed and move around on his own [4]. The primary disadvantage of this design is that it is prone to falling over. The large height and relatively small base make it somewhat unstable [4].

\section{Dyaun intravenous poles}

A more advanced free-standing IV pole is Dyaun IV pole shown in Figure 1c. Although this stand is not utilized nowadays, it does offer a glimpse of a better IV stand design. This design offers a simplified height adjustment, away to organize tubes and wires, wheel brakes, a handle and an overall more pleasing appearance and feel [5,21]. However, some drawbacks of the simple IV pole apply to this design; primarily the possibility of falling over, and its cost [4].
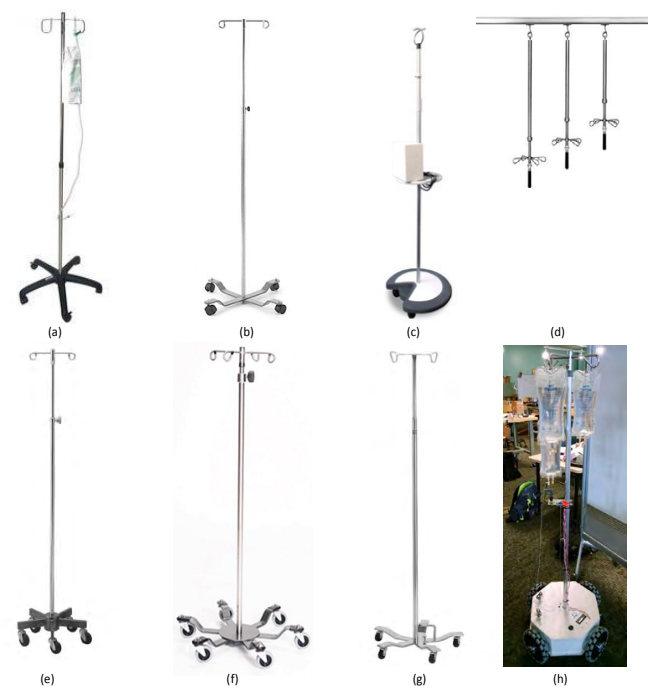

Figure 1: The existing Intravenous (IV) poles. (a) The first simple free standing designs. (b) The second simple free standing design. (c) Dyaun IV Pole. (d) Hanging IV Stand. (e) Lakeside Chrome Infusion Pump Pole. (f) 6 Leg Infusion Pump Intravenous Pole. (g) 5 Leg Infusion Pump Intravenous Poles. (h) Robotic Stand.

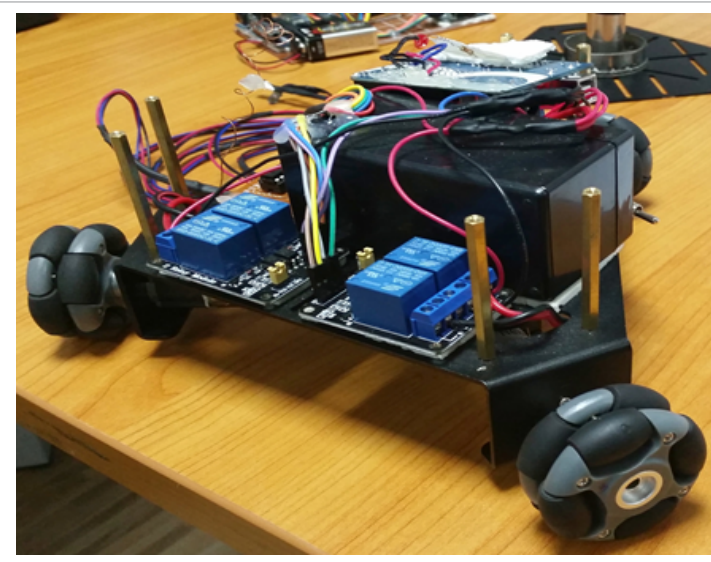

Figure 2: The Robotic Mechanical Base Description, Omni Wheel Chassis Holding DC Motors.

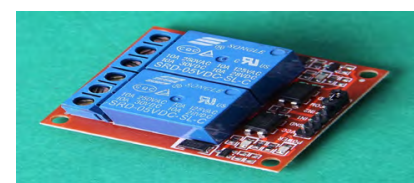

(a)

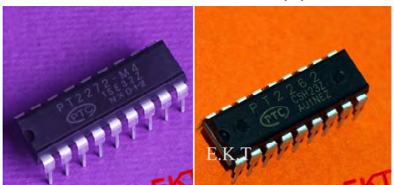

(b)
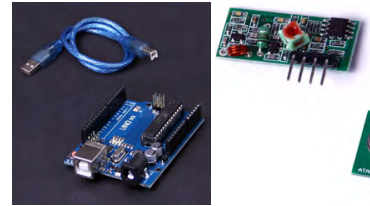

(e)

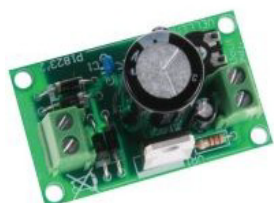

(b)

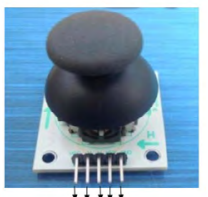

$(\mathrm{d})$

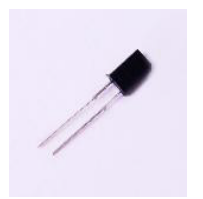

(f)
Figure 3: Robotic Electrical and Electronic Components. (a) Arduino Module Relay SRD 5 VDC-2 Channels. (b) DC-DC electronic converter. (c) Decoder and Encoder. (d) Joystick Drive. (e) Arduino UNO, Receiver module (Rx) and Transmitter module (Tx), respectively. (f) Photodiode Utilized for the Alarm System.

\section{Hanging intravenous poles}

Hanging IV pole, is another design for IV stands Figure 3 (d). They are called so because they are attached to the roof, and directed through the patient bed.

A drawback of these IV stands is that they do not allow the mobilization, so whenever the patient needs to move, someone should hold his IV bags [4].

\section{Lakeside chrome infusion pump poles}

The Infusion Pump stand is one of the old invented poles [22]. The lakeside chrome infusion pump (6 Leg pole and 4 Ram's Horn Hook) is another common IV pole in the medical field see Figure 1 (e). The bottom pole is $11 / 4$ " diameter chrome plated steel tubing and the top pole is $7 / 8$ " in diameter. The 6-leg oversize 26 " base offers exceptional stability for heavy duty use. The rubber wheels and casters provide quiet and effortless movement allowing easy mobility. The height adjustment 
range for this particular stand is 52.25 " to 102.75 " [4,11].

\section{Other designs of infusion pump intravenous poles}

A 6 Leg, 24" diameter with Stainless Steel Pole and Low-Profile Base Figure 1 (f). 1" diameter Stainless Steel pole adjusts 50" to 92". It includes a "no lose" friction knob and a base weight of $23 \mathrm{lbs}$, and includes 3" scuff resistant casters [11]. Another type is the $5 \mathrm{Leg}, 22$ " diameter with Stainless Steel Pole and Low-Profile Base Figure 1 (g). 1" diameter Stainless Steel pole adjusts 50" to 92". It includes a "no lose" friction knob, its base weighs $22 \mathrm{lbs}$, and it includes 3 " scuff resistant casters [11].

\section{Robotic Stand}

An autonomous IV stand showcased in Figure 1 (h) is a device that allows for mobile medicine distribution without the need for the patient to maneuver the device. One of the drawbacks of this system is the weight that it can handle [9]. All the types of the Intravenous stands discussed vary with respect to the structure, size, and number of hooks.

\section{Novel Robotic IV Pole}

As until today there is no design for IV stands that take into consideration high mobility and feasibility of use, in addition to automating the detection level of fluids in the IV bags, a new IV pole was designed. We developed this pole and named it the "Robotic IV Pole". This pole is (i) designed carefully, (ii) implemented in a professional way and (iii) matches the required safety. The former and latter steps are described in details in this section.

\section{Robotic IV Pole Design}

Herein the essential components of the developed robot are listed with emphasis on their essential role and function.

Wheels and Motors: In order to design a robotic IV pole capable of moving in any direction and rotating at any angle $\theta$, omni wheels have been used instead of regular wheels as shown in Figure 2. Figure 2 (a) represents a single omni wheel and Figure 2 (b) showcases the number as well as the placement of these wheels. These wheels can roll normally like any other wheel or it can roll laterally using the additional wheel parts along its circumference.

The advantage of omni wheel over standard wheels is its ability to move in any direction without the need to a traditional swivel mount [23]. Omni wheel is a $48 \mathrm{~mm}$ wheel that works independently in order to improve the control and permit the rotation and side-way movements in confined areas [24]. There is no preference for the omni wheel's orientation when it is meant to move forward, backward, to the left, to the right, or it is meant to rotate [25]. In our study, this wheel weighs $40 \mathrm{~g}$ and possesses a load capacity of $2 \mathrm{~kg}$, to benefit from its small dimension and excellent specifications.

Three 12 VDC motors providing a power of $6 \mathrm{~W}$ and a load speed of 120 RPM [26] were used. All motors were placed in an aluminum triangular chassis (Figure 2b). This forms the base of our robotic IV pole.

Relay: The Relay is composed of a coil that expands whenever the voltage reaches to it. Each Relay contains 2 contacts, this contact works as a switch. To drive the Relay, these 2 contacts must close on each other, and this happens if one of the contacts is maintained closed and the other one is opened, and vice versa. However, if the 2 contacts are opened or closed simultaneously, they will not close on each other, and the relay will not function. To keep the contact closed, we assign a
"LOW" or "0" in the Arduino code, and to open it we assign a "HIGH" or " 1 " in the Arduino code.

The Arduino Module Relay utilized in the project is shown in Figure 3 (a). Figure 3 (a) showcases the Arduino Module Relay SRD 5VDC-2 Channels. It is characterized with its high pull and with LED indicator that lights after the power is ON. This Relay was utilized in our system's design in order to provide more control on the DC motors and the omni wheels which are connected to the motors.

DC-DC electronic converter: The DC-DC converter, shown in Figure 3 (b), is an electronic device that converts a source of Direct Current (DC) from one voltage level to another. It is a type of electric power converter [27] utilized in our design to transform the 12 VDC battery to 5 VDC. This is because the Arduino Relays are supplied with $5 \mathrm{~V}$ and cannot handle the $12 \mathrm{VDC}$ supply.

Encoder and decoder: An Encoder based on CMOS technology was utilized to transfer the data from the Ar- duino to the Radiofrequency (RF)/Transmission (Tx), RF/Tx, since the Arduino cannot be connected directly to the RF/Tx. A Decoder, based on CMOS technology, was also utilized in designing our robotic base. The Decoder was utilized to transfer the data from the $\mathrm{RF} / \mathrm{Rx}$ on the receiving side, and send it to the Arduino, since the Radio-frequency/Receiver $(\mathrm{Rx}), \mathrm{RF} / \mathrm{Rx}$ cannot be connected directly to the Arduino. In both the Encoder and the Decoder (Figure 3c) left and right, respectively), data bits pins were employed.

Joystick: Arduino joystick controller module is an electronic component with a small size of $3.2 \times 3.5 \mathrm{~cm}$ Figures $3(\mathrm{~b})$ and $3(\mathrm{~d})$. This joystick was utilized to act as a potentiometer of $10 \mathrm{k} \Omega$ resistance [28].

As the joystick is altered by the finger in different directions, the resistance will change. When the joystick is pressed along the direction of the arrow, the voltage reading increases up to $5 \mathrm{~V}$; however, when the joystick is pressed opposite to the arrow, the voltage reading reduces to a minimum of $0 \mathrm{~V}$. When the joystick is pushed in, the robotic IV pole rotates. The joystick component is meant to serve as a preliminary remote control for our robot.

Micro-Controller and Wireless System: The micro-controller comprises the Arduino UNO, which is used to code both the transmitted and received data through binary numbers. This platform is based on the ATmega328 micro-controller. This micro-controller contains digital input/output pins, analog inputs, an oscillator, a USB connection, a power jack, an In-Circuit Serial Programming (ICSP) header and a button. Of all the preceding boards, the UNO used in our system does not utilize the FTDI USB-to-serial driver chip [29].

Two Arduino Uno boards and a $T x / R x 315 \mathrm{MHz}$ module, shown in Figure 3e) (left) were used and coded, in order to link the data between the transmitting side and receiving side. The Arduino Module $T x / R x$ is characterized with its strong anti-interference, high reliability and stability, long life, low power consumption and it is similar to a remote control, i.e., does not have any interference garbled phenomena. And the transmitted signal to the wireless receiver can cross walls [4]. This Arduino Module was utilized to send data from the transmitter side to the receiver side through $315 \mathrm{MHz}$ frequency and by an antenna connected to each of the $\mathrm{RF} / \mathrm{Tx}$, and $\mathrm{RF} / \mathrm{Rx}$, with a $23.5 \mathrm{~cm}$ length according to the lambda rule:

$$
\lambda=c . f,
$$

where $\lambda$ is the wavelength in $(\mathrm{m}), c$ is the speed of light in vacuum $(\mathrm{m} / \mathrm{s})$ and $f$ is the frequency in ( $\mathrm{Hz})[30]$. As shown in Figure 3e) (right), the 
Citation: Sayed-Kassem A, Ghandour A, Hamawy L, Zaylaa AJ (2017) Cutting-Edge Robotic Intravenous Pole: Preliminary Design and Survey in Academic Medical Center in Lebanon. J Biomed Eng Med Devic 2: 124. doi: 10.4172/2475-7586.1000124

Page 4 of 9

transmitter module has 3 pins, 2 for the power supply $5 \mathrm{~V}$ and one Data pin connected to the Encoder. Furthermore, the receiver module has 4 pins, 2 for the power supply of $5 \mathrm{~V}$, and 2 Data pins connected to the Decoder.

Photodiode: The Silicon Pin photodiode, shown in Figure $3 \mathrm{f}$ or BPW41 utilized in this work, is a high speed and high sensitive Pin photodiode. It functions on a threshold, such that whenever the threshold, which acts as sensitivity, increases there will be an output depending on what is coded on the Arduino.

Robotic IV Pole Implementation: The block diagram showcasing the data acquisition and transmission of the novel IV pole is detailed in Figure 4. Note that the electronic components and programming tools that were used in the implementation steps are reported in Table 1.

All the upcoming steps required to function the system are coded using the micro- controller discussed in section 3.1.6. This is achieved using the following two flowcharts in Figure 5. Figure 5 (a) showcases the flowchart of the Arduino UNO of the transmitting side, and Figure 5 (b) showcases the flowchart of the Arduino UNO of the reception side. The robotic IV pole implementation (Figure 6) comprises a summary of the methodology of implementation and function of our robotic IV pole. It is divided into three essential parts:

\section{Insert Table 1 Here}

1. Building the joystick to trigger the Robot to handle large weight;

2. Building the robotic base and connecting it to the stand to achieve balance and safety;

3. Building the alarm system on the IV bag where the medications are hung.

The steps for installing the first building block (part 1) which is the joystick are given as follows:

On the transmitting side:

- Connect the joystick pins X, Y, Z to the Arduino analog inputs A0, A1, A2 respectively.
- Ensure that the module contains 5 pins, 2 of them for a power supply $(5 \mathrm{~V})$. However, 2 pins: $\mathrm{X}$ and $\mathrm{Y}$, respectively, read a voltage of $c 2.5 \mathrm{~V}$. On the transmitting side, to build the encoder connection:

- $\quad$ Supply the encoder by $5 \mathrm{~V}$ from the Arduino board.

Connect three resistors of $10 \mathrm{k} \Omega$ between the pins D3 (pin number $10), \mathrm{D} 2$ (pin number 11), D1 (pin number 12) of the encoder and their soldering respectively.

- Connect a 3.3 $\mathrm{M} \Omega$ resistor between osc1 and osc2 of the encoder.

- Connect the digital output 8,9 and 10 of the Arduino to the digital data bit D3, D2, D1 of the encoder respectively.

- Connect the Encoder pin Dout is to the Data pin of the $R F / T x$ module.

- Measure the length of the Antenna of the $R F / T x$ to be at $23.5 \mathrm{~cm}$.

- Finally, place all the components with their connections on a Printed Circuit Board (PCB).

The steps required for installing the second building block (part

(2)) i.e., robotic IV pole's base are as follows:

- Use omni wheels instead of regular wheels to build the robotic mechanical part.

- Use three $12 \mathrm{VDC}$ motors to transform the electrical energy into a mechanical energy.

- Connect the three 12 VDC motors to an omni wheel to form the base of the robotic IV Pole.

- Connect this base to a micro-controller (Arduino UNO) to program it.

- Connect the IV stand, which is extendable in elevation, to the base. On the reception side, to build the Decoder and RF/Rx connections:

- $\quad$ Supply the Arduino by a 12 V DC battery.

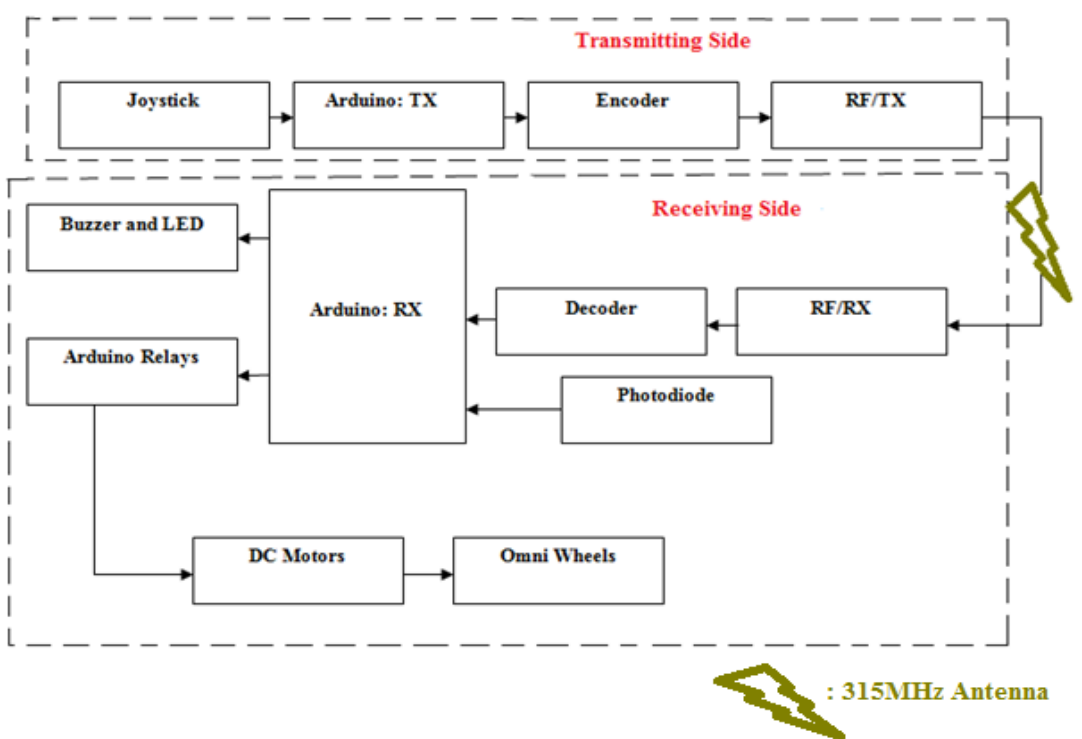

Figure 4: The Block Diagram of the Novel Robotic Intravenous Pole. 
On Transmission Side

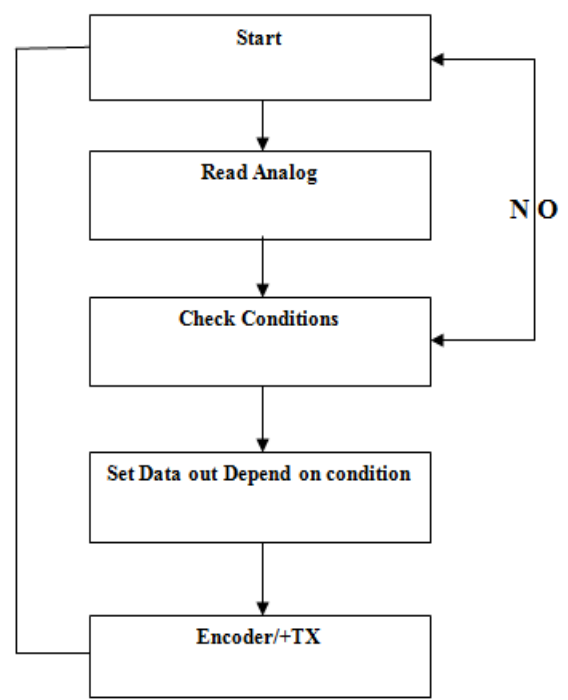

Figure 5: The Flowchats of the Arduino UNO. (a) The Transmission Part. (b) The Reception Part.
On Reception Side

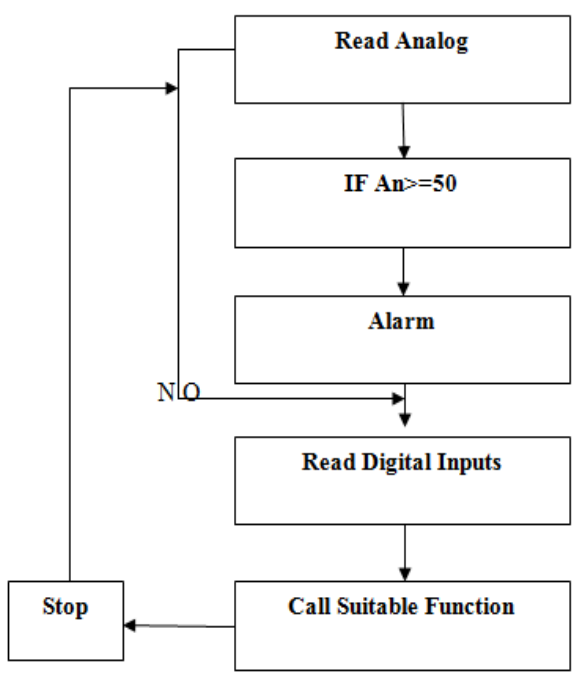

IV Stand Robot Design

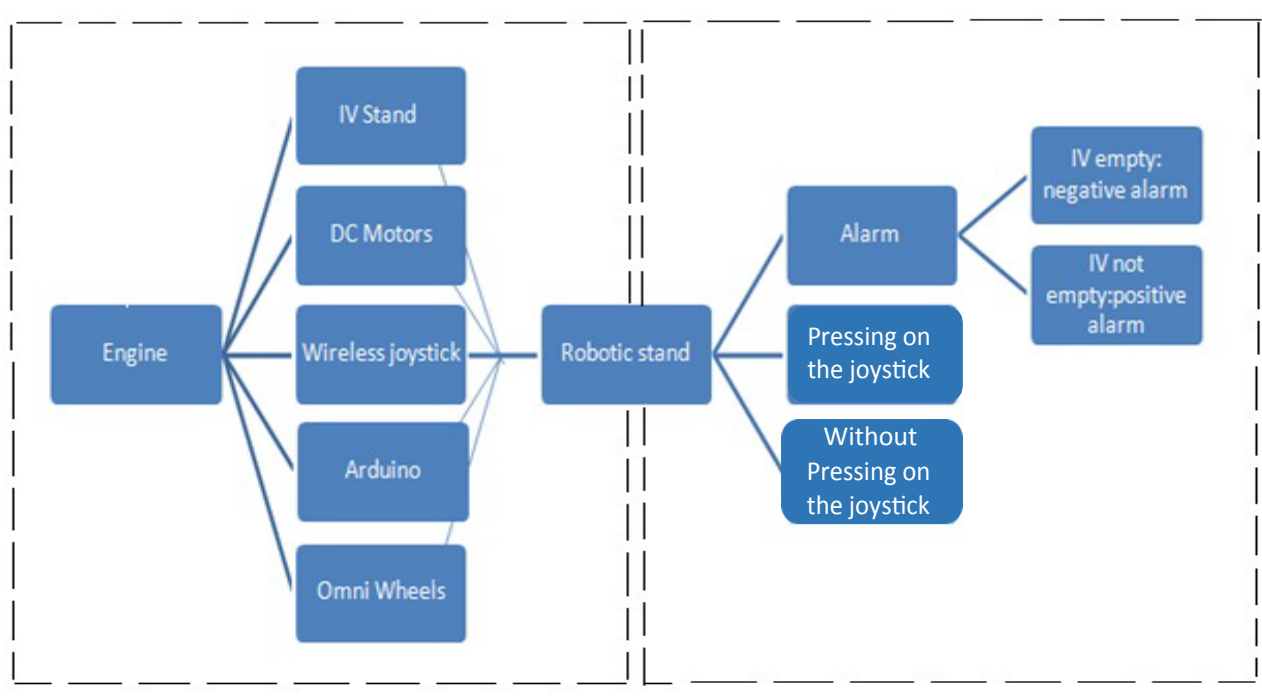

Figure 6: The Robotic Intravenous Pole Implementation.

- $\quad$ Supply the decoder board by $5 V$ from the Arduino (Vcc, Gnd) through Vin, Gnd pins.

- $\quad$ Provide the $R F / R x$ module by a $5 V$ from the Arduino (Vcc, Gnd) through the pins Vin, Gnd.

- Connect the data pin of the RF/Rx module to the data in pin of the decoder.

- $\quad$ Connect the decoder pins D3, D2, D1 and VT to the Arduino pins $8,9,10$ and 11 , respectively.

- Connect a $10 \mathrm{k} \Omega$ resistor between osc1 and osc2 pins of the decoder.
The steps required for installing the third building block (part (3)) which is the Alarm system of the IV bag are as follows:

- Connect the photodiode to the Analog input of the Arduino A0.

- Use this photodiode by connecting it to the coded Arduino on the receiving side.

- Connect the LED to the digital input of the Arduino 13.

- Connect the Buzzer to the digital Input of the Arduino A1.

- Set a threshold 50 through an "If statement" in the Arduino code.

- Number 50 is selected from empirical inference, thereby whenever the threshold is higher than 50 in the Analog input the photodiode 
Citation: Sayed-Kassem A, Ghandour A, Hamawy L, Zaylaa AJ (2017) Cutting-Edge Robotic Intravenous Pole: Preliminary Design and Survey in Academic Medical Center in Lebanon. J Biomed Eng Med Devic 2: 124. doi: 10.4172/2475-7586.1000124

Page 6 of 9

\begin{tabular}{|c|c|}
\hline Electric Components & Quantity \\
\hline 12 V DC Motors, Omni Wheels, LEDs & 3 from each \\
\hline Arduino Joystick Module & 1 \\
\hline Arduino Uno Atmega 328P-PU & 2 \\
\hline PT2262 Encoder and PT2272 Decoder & 1 from each \\
\hline Arduino Module TX/RX 315MHz & 3 \\
\hline 12 VDC Battery and 9 VDC Battery & 1 from each \\
\hline Resistors & 9 \\
\hline Photodiode & 1 \\
\hline PCB Boards and ON/OFF switch & 2 from each \\
\hline
\end{tabular}

Table 1: A Summary of the Electronic Components Utilized in Robotic IV Pole Design.

is connected to, a buzzer will beep, and LED will light as a result of the digital output. The buzzer, and a LED will be ON through their connections with the digital output of the Arduino. Ensure that as the IV fluid runs away and the IV bag becomes empty, the two arms of the photodiode will sense and induce a sound and a red light.

\section{Robotic IV pole safety}

Taking into account the mechanical influence and safety of our developed system, the study of the balance of the stand was essential. The novel IV pole has to be capable of moving electronically while holding different weights by analogy to other studies [25]. For this purpose, it is crucial to:

- Use stand with extendable height;

- Add small metallic accessories to fix the photodiode on it; Place a metallic spring between the base of the robot and the extendable stand, to provide safety for the design and to ensure the balance.

- Release the spring to recover the classical pole design if a sudden electronic problem has occurred to the robotic base.

\section{Robotic IV Pole Evaluation and Survey}

In order to evaluate the performance of the developed Robotic IV Pole, a purely prospective study was applied. A questionnaire has been prepared and a survey was done.

The questionnaire shown in Figure 7 provides the main questions on the comfort, feasibility and cost of the Robotic IV pole as opposed to alternative poles. The survey was carried out on $N=108$ personnel, including 36 patients, 36 nurses and 36 hospital Human Resources in Lebanon.

\section{Experimental Results}

The experimental results are divided into 3 parts, the mobility capabilities of the Robotic IV Pole, the weight tolerance of the Robotic IV Pole and the Alarm system capabilities associated with the Robotic IV Pole.

\section{IV pole Displacement/Mobility Results}

Figure 8 showcases the resulting Robotic IV Pole. To test the performance of this novel robotic IV pole, the pole was put in use by a group of patients. When the patient's triggered the joystick on the transmitting side forward, backward, left or right using their fingers, the robotic IV pole (receiving side) responded by moving to the same direction using two wheels. Furthermore, when the patient pushed in they key button, the robot rotated using the 3 wheels.

The joystick provided a displacement along $x, y$ and $\theta$ directions,

where:

- The positive $x$ is the forward displacement, and the negative $x$ is the backward displacement.

- The positive $y$ is the left direction, and the negative $y$ is the right direction.

- The $\theta$ or inward pressing of the key, is the rotation about the $\mathrm{z}$-axis.

When the displacement along the positive $\mathrm{x}$, negative $\mathrm{x}$, positive $\mathrm{y}$ or negative direction is triggered, only 2 motors work triggering $2 \mathrm{omni}$ wheels to move. Moreover, when the rotation is triggered about the $\mathrm{z}$-axis, the 3 motors work, causing the 3 omni wheels to move.

Experimental testing reflected that the wireless communication between the patient and the stand is not altered and withstands a distance $\geq 30 \mathrm{~m}$.

\section{The IV Bag Weight Results}

Results showed that the novel robot is capable of holding a weight up to $15 \mathrm{~kg}$. This is the weight below which the robotic IV pole is maintained balanced.

\section{The Alarm System Results}

On the other hand, the alarm system was tested after utilizing different medications and saline bags. When the fluid in the IV bag runs out, the sensitivity across the photodiode increases. As this photodiode is connected to the analog input of the Arduino UNO, and when the threshold is less than 50 (the empirical threshold) the buzzer beeped and the LED lit showing that the fluid bag is emptied.

\section{Robotic IV Pole Survey Results}

Figure 9 showcases the variation of the number of nurse's responses with respect to the average degree of agreement and benefit of the

\section{ROBOTIC IV POLE SURVEY FEEDBACK QUESTIONNAIRE}

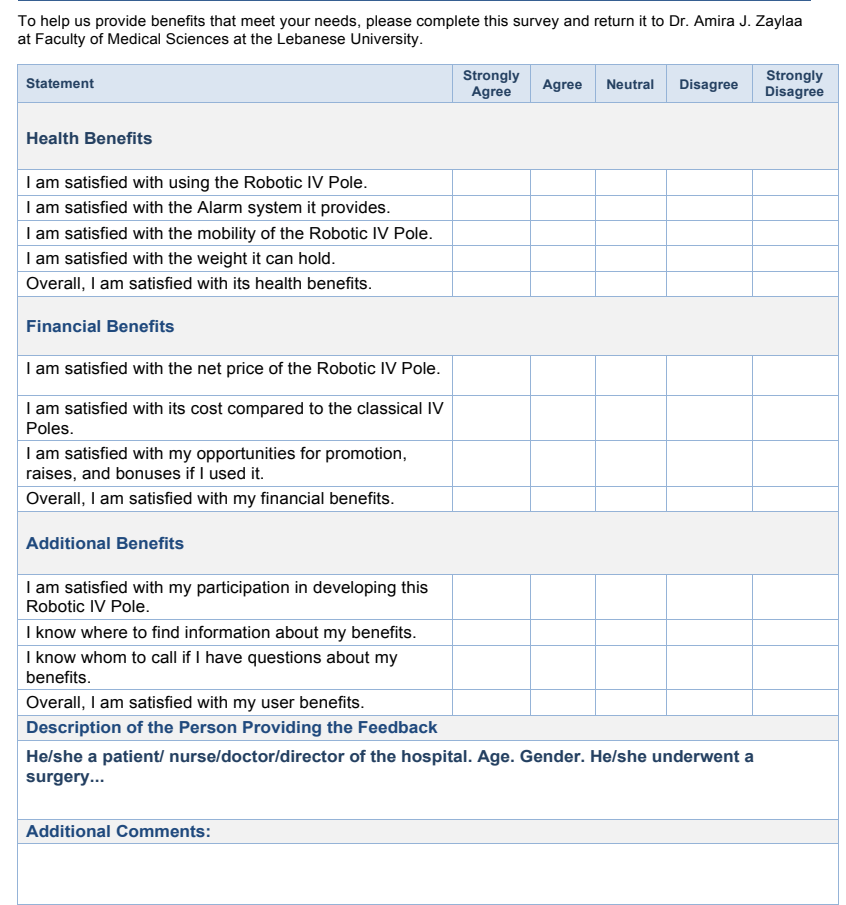

Figure 7: The Questionnaire of the Robotic IV Pole Survey. 
Robotic IV Pole Technology demo. Of all the nurses who participated in the survey and answered the questionnaire, the majority 'agreed' on the health benefits (coded in dark blue), financial benefits (coded in green) and additional benefits (coded in yellow). Table 2 reports the avraged survey results of the preliminary Robotic IV pole design. More specifically as inferred from Table $2,87.55 \%(27.78 S A+59.77 A$ from Figure 9) agreed on the health benefits, $61.79 \%(13.19 S A+48.6 A)$ agreed on the 'financial benefits', and finally $91.66 \%(26.39 S A+65.27 A)$ agreed on the 'additional benefits'. Among the vast benefits agreed on, the utmost benefit was that of the additional benefits (including the maintenance services and the service the guides the Robotic IV pole user and answer his questions etc), followed by the Health benefits, and finally the financial benefits.

Figure 10 showcases the number of patient's responses as a function of the average degree of agreement and benefit of the Robotic IV Pole Technology demo. Of all the patients who participated in the survey, the majority 'agreed' on the health benefits (coded in dark blue), financial benefits (coded in green) and additional benefits (coded in yellow). More specifically, Table 2 reports that $94.60 \%(46.91 S A+47.69 A$ from Figure 10) agreed on the health benefits, $88.45 \%(49.03 S A+39.42 A)$ agreed on the 'financial benefits', and finally $93.26 \%(43.27 S A+49.99 A)$ agreed on the 'additional benefits'.

Figure 11 reveals the number of HR's responses with respect to the average degree of agreement and benefit of the Robotic IV Pole Technology demo. Of all the HRs who participated in the survey, the majority 'agreed' on the health benefits (coded in dark blue), financial benefits (coded in green) and additional benefits (coded in yellow). According to Table 2, $87.55 \%(27.78 S A+59.77 A$ agreed on the health benefits, $61.79 \%(13.19 S A+48.6 A)$ agreed on the 'financial benefits', and finally $91.66 \%(26.39 S A+65.27 A)$ agreed on the 'additional benefits'

\section{Discussion}

Survey Results on the nurse's population showed that the highest benefit went to the additional benefits (including the maintenance services and the service that guides the Robotic IV pole user and answer his questions etc). Additional benefits are followed by the Health benefits, and finally the financial benefits. This is due to the fact that the maintenance and training services $(91.66 \%$ set in bold in Table 2$)$ are of paramount interest for the nurses. In addition to the health benefits from the alarm to the patient-dependent mobility of the Robotic IV Pole, as it does not depend on the nurse and reduces his/her time elapsed by each patient.

Results on the patient's population associated the highest benefit to "health" followed by the "additional benefits" and finally to the "financial" ones. This is due to the fact that the health benefits (94.60 $\%$ set in bold in Table 2) are the primary thing that concerns patients, especially elderly ones who are incapable of maneuvering the IV pole or those going out of a surgery.

Of all the population that we have explored, the majority gave more value to the health and alarm benefits as compared to the financial benefits. Knowing that an average of $93.26 \%$ at least agreed on the estimated price of the novel system. When exploring the Human Resources (HRs) and doctors, the statistical results associated the highest benefit percentages to the "health" followed by the "additional benefits" and finally to the "financial" ones. This is due to the fact that the health benefits are the primary thing that concerns patients especially elderly ones who are incapable of maneuvering the IV pole or those

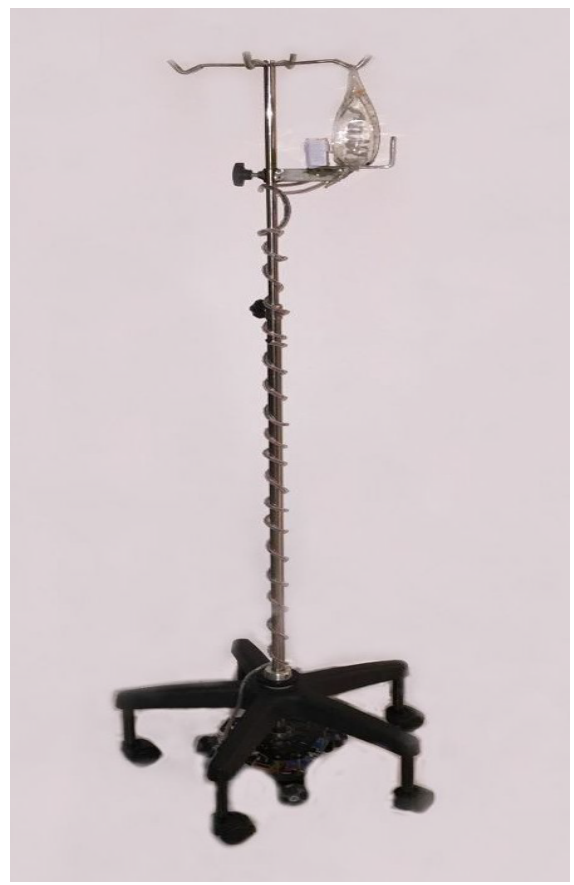

Figure 8: The Novel Robotic IV Pole.

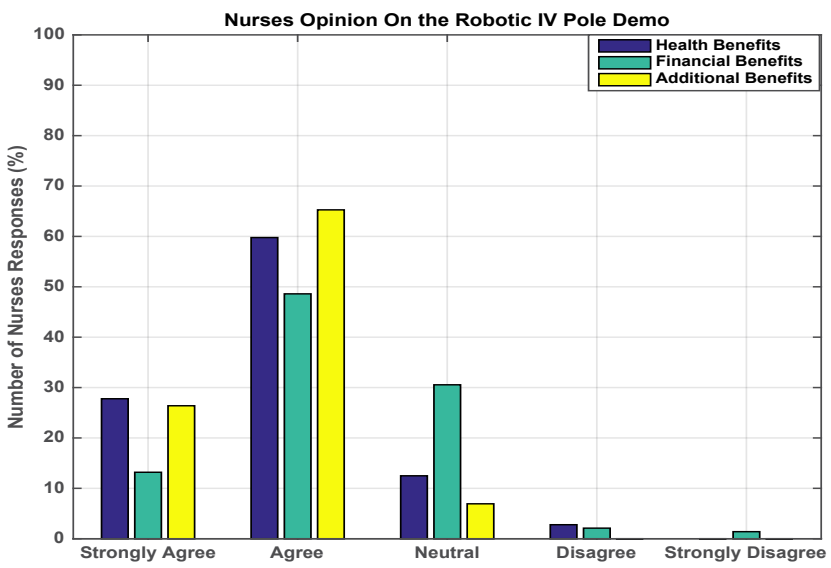

Figure 9: The Nurse's Feedback on the Robotic IV Pole Demo.

going out of a surgery. Of all the population that we have explored, the majority gave more value to the health and alarm benefits as compared to the financial benefits, when on average $67.50 \%$ at least agreed on the estimated price of the novel system. Results also showed that the HR's and Hospital Managers were ready to afford this new technology. This was highlighted when $61.79 \%$, as an average value, at least 'agreed' that the estimated price of the technology embedded in the individual Robotic IV Pole is less than that of the IV Poles present in the market or under research. Besides, $87.55 \%$ of the volunteers strongly agreed on the feasibility in maneuvering the novel Robotic IV, and on the weight that the Pole can withstand. Of all the 108 personnel studied in the academic medical center in Lebanon, most at least 'agreed' on the Alarm system and some suggested that it could be further improved.

This novel robot attained its role in making the patient feel more comfortable. After the development of this novel robot, the patient 


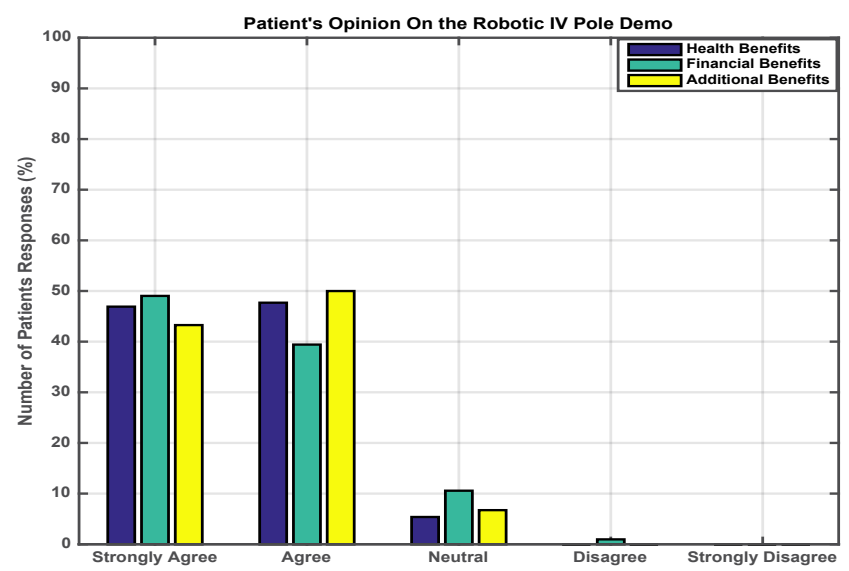

Figure 10: The Patient's Feedback on the Robotic IV Pole Demo.

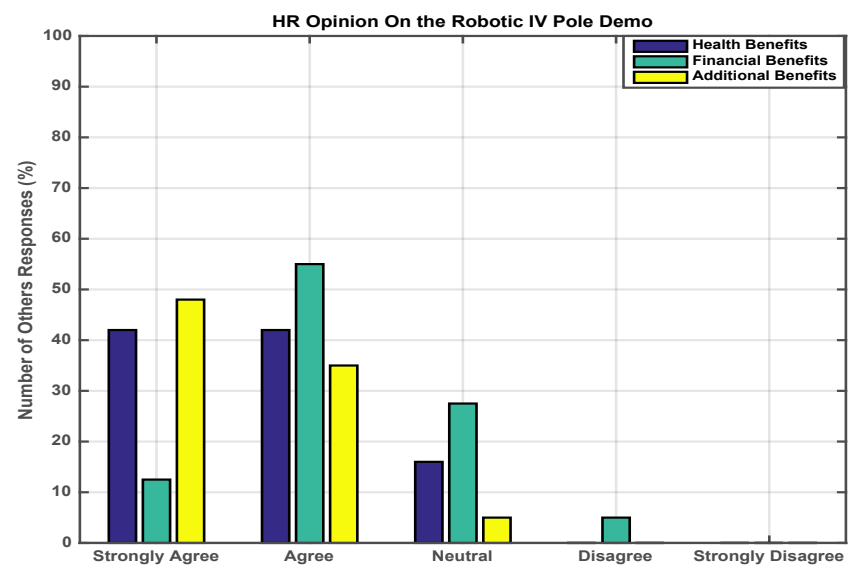

Figure 11: The Human Resources Feedback on the Robotic IV Pole Demo.

\begin{tabular}{|c|c|c|c|}
\hline Type of Personnel Benefits & Health (\%) & Financial (\%) & Additional (\%) \\
\hline & SA-A & SA-A & SA-A \\
\hline Nurses & 87.55 & 61.79 & $\mathbf{9 1 . 6 6}$ \\
\hline Patients & $\mathbf{9 4 . 6 0}$ & 88.45 & $\mathbf{9 3 . 2 6}$ \\
\hline Human Resources & $\mathbf{8 4 . 0 0}$ & 67.50 & 83.00 \\
\hline
\end{tabular}

Table 2: The Averaged Survey Results of the Preliminary Robotic IV Pole Design.

can feasibly maneuver his IV stand using the joystick as opposed to the Simple Free Standing IV Pole, Dyaun IV Pole, Hanging IV Pole, Lakeside Chrome Infusion Pump Pole and other Designs of Infusion Pump IV Pole $[4,11,21]$. Pryor et al. and Vingnali et al. provided a manual IV-pole maneuvering by the patient/nurse [3,4]. In contrary, the degrees of freedom (variables) of our Robotic IV-pole are driven electronically. The preliminary remote system, i.e., joystick and the robotic base, provided the novel system with 3 degrees of freedom ( $\mathrm{x}$, $\mathrm{y}, \theta)$.

Schott et al. presented the completely balanced free IV stand assembly [5]. As per Schott et al. our robotic system was balanced and mounted with a spring, making the recovery of the classical IV pole possible when needed. Based on what preceded, one of the important findings of the robotic IV pole is that it can handle the degrees of freedom provided by the manual drive, as well as the 3 degrees of freedom provided by the electronic and wireless drive.
The robotic IV stand performed successfully not only from the electronic/communication perspective, but also from the mechanical perspective, through making the IV stand hold tight to the base accomplishing the biomedical engineer purposes. Thereby the mechanical safety was taken into account to preserve the balance of the robot during movement.

Despite Binger et al. and Ciullo reported theuseof IV robots for biomedical purposes [9,31-33], they showed weight limitations and lacked the alarm system. Our robotic IV-pole was designed to overcome this limitation and was associated with an alarm system.

\section{Conclusion}

A cutting edge robotic IV pole was developed, successfully maneuvered and underwent a statistical survey on 108 personnel from Lebanese population. The population was divided into 3 groups according to their profession.

Survey results revealed that $100 \%$ of the people were volunteering to facilitate the progress of the research and development of our Novel Robot. Survey results on the health, financial and additional benefits of the Robotic IV Pole showcased that the 'Agree' feedback was exterior to $80 \%$ in the explored Lebanese population. The novel robotic IV pole is maneuvered electronically by the nurse or escorts, since the robotjoystick communication holds for a large range. Another crucial finding of this development is reflected by the capability of the novel robot to hold a weight up to $15 \mathrm{~kg}$ while maintained balanced. Furthermore, wireless communication was possible due to the use of Radio frequency and an antenna.

As per any novel technology, there are some electronic drawbacks as a perspective we have few recommendations that have to be considered.

\section{Recommendations}

For developing the system, we propose the following:

- The use of a weight sensor instead of the photodiode in the mounted alarm system.

- Transforming the current joystick into an advanced drive though automating the Robotic IV Pole.

- Improving the drive of motors between the transmitting side of the Robotic IV pole, and the receiving side.

- Add the option of obstacle detection to our robotic IV pole as discussed recently in $[32,33]$.

\section{Acknowledgements}

The authors acknowledge the Lebanese International University in Beirut for supporting this work. Authors would like to thank Miss Alaa Zaylaa for her valuable feedback and Miss Sara Abou Diab for helping us in the survey.

\section{Conflict of Interest}

The authors declare that there is no conflict of interest regarding the publication of this paper.

\section{References}

1. Jost G, Tseng CC (1990) Baxter International Inc., assignee. Home care intravenous stand. United States Patent US 4,905,944.

2. Larson GR (1980) Larson Godfrey R, assignee. Handle for mobile intravenous stand. United States Patent US 4,225,104.

3. Pryor JW (1982) Pryor John W, assignee. Ambulatory patient support stand United States Patent US 4,332,378. 
Citation: Sayed-Kassem A, Ghandour A, Hamawy L, Zaylaa AJ (2017) Cutting-Edge Robotic Intravenous Pole: Preliminary Design and Survey in Academic Medical Center in Lebanon. J Biomed Eng Med Devic 2: 124. doi: 10.4172/2475-7586.1000124

4. Vignali M (2006) Intravenous Stand Design. Project partners from Huazhong University of Science and Technology, China.

5. Schott JC, Schott MD, Fox JD (2000) Schott Jeffery C, Marc D, Fox Jeffrey $\mathrm{D}$, assignee. Intravenous stand support assembly. United States Patent US $6,079,678$.

6. Adelman GZ (2006) Adelman Gregg Z, assignee. Transportable intravenous bag stand. United States Patent US 6,983,915

7. Wilt Jr CF (1985) St. Joseph's Hospital, Medical Center, assignee. Apparatus for facilitating intravenous feeding during transportation of patient. United States Patent US 4,511,157.

8. Qureshi MO, Syed RS (2014) The impact of robotics on employment and motivation of employees in the service sector, with special reference to health care. Safety and Health at Work 5: 198-202.

9. Binger M, Conway C, Goddard N, Jacobs N, Pysher C, et al. Project Number: P15073 Autonomous IV Stand.

10. Wiederhold BK, Riva G (2009) Affective robot for elderly assistance. Annua Review of Cybertherapy and Telemedicine 2009: Advanced Technologies in the Behavioral, Social and Neurosciences 144: 44

11. Michael M (2012) Arduino Cookbook.

12. Bancalari CG (1988) Icu Medical, Inc., assignee. Collapsable pole and stand combination. United States Patent US 4,744,536.

13. Gawlik GM, Hoyt RD, Anderson EA (1989) Portable intravenous stand. United States Patent US 4,807,837.

14. Lafferty WG, Howard RE (1990) Polymedical Technologies, Inc., assignee. Fully portable medical IV equipment stand/pole. United States Patent US $4,892,279$.

15. Pryor JW, Pryor JW, Ratcliff JW (1994) Pryor Products, assignee. Multi-pole support stand. United States Patent US 5,344,169.

16. Woodward J (1995) Woodward, John, assignee. Portable intravenous support stand. United States Patent US 5,458,305.

17. Phillips JR, Phillips JF (1999) Phillips James R, Janice F, assignee. Support cart apparatus for supporting intravenous fluid dispensing systems. United States Patent US 5,857,685.

18. Elliott D, Morrow DL, Rubin L (2003) Emergent Innovations, LLC, assignee. Intravenous (IV) pole supporting systems. United States Patent US 6,619,599.

19. Tollefson J (2012) Clinical psychomotor skills: assessment skills for nurses PDF. Cengage Learning Australia.
20. Kunik BJ (2002) Pole apparatus for hanging intravenous bags or other similar devices. US Patent D457,239.

21. Kramer KL, Weismiller MW (1995) Hill-Rom Company, Inc., assignee. Sliding IV pole. United States Patent US 5,407,163.

22. Varga SI, Dougherty RL, Dandy DC, Thomas WJ (1985) Mt. Sinai Medical Center of Greater Miami, assignee. Intravenous infusion pole attachment United States Patent US 4,511,158.

23. Bemis S, Riess B, Nokleby S (2008) Control of a novel omni-directiona platform. In: Electrical and Computer Engineering, 2008. CCECE 2008. Canadian Conference on 2008 May 4, pp: 000761-000766.

24. Salih JE, Rizon M, Yaacob S, Adom AH, Mamat MR (2006) Designing omnidirectional mobile robot with mecanum wheel. American Journal of Applied Sciences 3: 1831-1835.

25. Wu Y, Chen K, Fu C (2016) Effects of Load Connection Form on Efficiezancy and Kinetics of Biped Walking. Journal of Mechanisms and Robotics 8: 061015

26. Ma X, Jiao Z, Wang Z, Panagou D (2016) Decentralized prioritized motion planning for multiple autonomous UAVs in 3D polygonal obstacle environments. In: Unmanned Aircraft Systems (ICUAS), 2016 International Conference on 2016 Jun 7, pp: 292-300

27. Yokomizo I, Horiuchi S, Matsumae M (2002) Rohm Co., Ltd., assignee. DC/DC converter. United States Patent US 6,400,211

28. Huzij R, Spano A, Bennett S (2013) Modern Diesel Technology: Heavy Equipment Systems, Modern Diesel Technology, Cengage Learning.

29. Panagou D (2015) Distributed coordination protocols for aggregation and navigation in multi-agent systems under local directed interactions. In: Decision and Control (CDC), 2015 IEEE 54th Annual Conference on 2015 Dec 15, pp: 2780-2785.

30. Bushong SC (2013) Radiologic science for technologists: physics, biology, and protection. Elsevier Health Sciences.

31. Ciullo SJ (2013) Making the switch to robotic iv preparation. Pharmacy Purchasing \& Products 10: 6

32. Kowalczyk W, Przybyla M, Kozlowski K (2017) Set-point Control of Mobile Robot with Obstacle Detection and Avoidance Using Navigation FunctionExperimental Verification. Journal of Intelligent \& Robotic Systems 85: 539-552.

33. Panagou D, Stipanović DM, Voulgaris PG (2016) Distributed coordination control for multi-robot networks using Lyapunov-like barrier functions. IEEE Transactions on Automatic Control 61: 617-632.
Citation: Sayed-Kassem A, Ghandour A, Hamawy L, Zaylaa AJ (2017) Cutting-Edge Robotic Intravenous Pole: Preliminary Design and Survey in Academic Medical Center in Lebanon. J Biomed Eng Med Devic 2: 124. doi: 10.4172/2475-7586.1000124
OMICS International: Open Access Publication Benefits \&

\section{Features}

Unique features:

- Increased global visibility of articles through worldwide distribution and indexing

- Showcasing recent research output in a timely and updated manner

- Special issues on the current trends of scientific research

Special features:

- $700+$ Open Access Journa

$50,000+$ editorial team

Rapid review process

Quality and quick editorial, review and publication processing

Indexing at major indexing services

Sharing Option: Social Networking Enabled

Authors, Reviewers and Editors rewarded with online Scientific Credits

Better discount for your subsequent articles

Submit your manuscript at: http://www.omicsonline.org/submission// 